\title{
Infection in Hypergraphs
}

\author{
Ryan Bergen* Shaun Fallat ${ }^{\dagger} \quad$ Adam Gorr $^{\ddagger}$ \\ Ferdinand Ihringer ${ }^{\S} \quad$ Karen Meagher ${ }^{\llbracket}$ Alison Purdy ${ }^{\|}$ \\ Boting Yang** Guanglong $\mathrm{Yu}^{\dagger \dagger}$
}

October 8, 2018

\begin{abstract}
In this paper a new parameter for hypergraphs called hypergraph infection is defined. This concept generalizes zero forcing in graphs to hypergraphs. The exact value of the infection number of complete and complete bipartite hypergraphs is determined. A formula for the infection number for interval hypergraphs and several families of cyclic hypergraphs is given. The value of the infection number for a hypergraph whose edges form a symmetric $t$-design is given, and bounds are determined for a hypergraph whose edges are a $t$-design. Finally, the infection number for several hypergraph products and line graphs are considered.
\end{abstract}

\section{Introduction}

The subject of zero forcing for graphs has been widely studied [1, 2, 6, 8]. In this paper we generalize the concept of zero forcing on graphs to hypergraphs.

In standard zero forcing for graphs, the vertices of the graph are coloured either black or white. A black vertex can force a white vertex to black according

\footnotetext{
*Department of Mathematics and Statistics, University of Regina, Regina, Saskatchewan, S4S 0A2. Email: bergen4r@uregina.ca.

${ }^{\dagger}$ Department of Mathematics and Statistics, University of Regina, Regina, Saskatchewan, S4S 0A2. Research supported in part by an NSERC research grant Application No.: RGPIN2014-06036. Email: shaun.fallat@uregina.ca.

${ }^{\ddagger}$ Department of Mathematics and Statistics, University of Regina, Regina, Saskatchewan, S4S 0A2. Email: gorr200a@uregina.ca.

$\S$ Department of Mathematics and Statistics, University of Regina, Regina, Saskatchewan, S4S 0A2 Research supported in part by a PIMS post-doctoral fellowship. Email: Ferdinand. Ihringer@gmail.com.

IDepartment of Mathematics and Statistics, University of Regina, Regina, Saskatchewan, S4S 0A2. Research supported in part by an NSERC research grant Application No.: RGPIN341214-2013. Karen. Meagher@uregina.ca.

I Department of Mathematics and Statistics, University of Regina, Regina, Saskatchewan, S4S 0A2. Email: Alison.Purdy@uregina.ca.

** Department of Computer Science, University of Regina, Regina, Saskatchewan, S4S 0A2. Research supported in part by an NSERC Discovery Research Grant, Application No.: RGPIN-2013-261290. Email: Boting. Yang@uregina.ca.

$\dagger^{\dagger}$ Department of Mathematics and Statistics, University of Regina, Regina, Saskatchewan, S4S 0A2 and Department of Mathematics, Yancheng Teachers University, Yancheng, 224002, Jiangsu, P.R. China. Research supported in part by an NSFC grant (No. 11271315), Jiangsu Qinglan Project (2014A). Email: yglong01@163.com.
} 
to a colour change rule. The colour change rule for standard zero forcing is that a black vertex can force an adjacent white vertex to black if it is the only white vertex adjacent to that black vertex. A set of vertices in a graph is a zero forcing set for the graph if when the vertices in this set are set to black and the colour changing rule is applied repeatedly, all the vertices of the graph are eventually forced to black. The zero forcing number of a graph is the size of the smallest zero forcing set for the graph. For a graph $G$, the zero forcing number is denoted by $Z(G)$.

The term "zero forcing" is based on an algebraic property of these sets. Consider a vector with the entries corresponding to the vertices of a graph. Further, assume the entries corresponding to a set of vertices in a zero forcing set for the graph are equal to zero. The zero forcing property of the set guarantees that such a vector is in the kernel of the adjacency matrix of the graph only if the vector is the zero vector. The term "zero forcing" refers to the fact that the remaining entries of the vector are forced to be zero for the vector to be in the kernel of the adjacency matrix.

For hypergraphs, there is no matrix analogous to the adjacency matrix of a graph and this notion of a set of entries in a vector forcing the other entries to be zero in a proposed null vector does not apply. However, in this paper we focus on generalizing the colour change rule and hence we use the term infection, rather than zero forcing. Terms such as infection, propagation, and searching have all been used for notions similar to zero forcing, see [7, 9, 10].

For infection in a hypergraph, the vertices are initially either infected or uninfected (as opposed to either coloured black or white, as they are in zero forcing for graphs). There is an infection rule that determines when vertices can infect other vertices (this is analogous zero forcing rule for graphs). In this case, it is a subset of infected vertices in an edge that may infect the remaining vertices in that specific edge, rather than a single vertex forcing another vertex. The following is the infection rule for hypergraphs.

Infection Rule: A non-empty set $A$ of infected vertices can infect the vertices in an edge $E$ if:

1. $A \subset E$, and

2. there are no uninfected vertices $v$, not contained in $E$, such that $A \cup\{v\}$ is a subset of an edge.

Similar to the case for graphs, if two vertices in a hypergraph are contained in a common edge, then we say that the vertices are adjacent. Further, for a hypergraph, we can define two sets to be adjacent if there is an edge that contains them both. So a set $A$ of infected vertices can infect an edge $E$ if there are no uninfected vertices outside of $E$ that are adjacent to $A$.

If $A \subset E$ satisfies the conditions set out in the infection rule, then we say that "the set $A$ infects the edge $E$ ". In the case of hypergraphs, it is an edge and all the vertices in the edge that are infected rather than a single vertex, as is the case for graphs. A set of vertices in a hypergraph is an infection set if when the vertices in the set are initially infected and the infection rule is applied repeatedly, then all the vertices in the hypergraph become infected. The infection number of a hypergraph $H$ is the size of a smallest infection set for $H$; the infection number of $H$ is denoted by $I(H)$. 
In a hypergraph the edges are subsets of the vertices, and can be of any size (the size of an edge is the number of vertices in the edge). If all the edges in a hypergraph contain exactly $k$ vertices, then the hypergraph is called a $k$ hypergraph. A 2-hypergraph is a graph; the infection number for a 2-hypergraph is equivalent to the zero forcing number of the graph.

Proposition 1.1. Let $H$ be a 2-hypergraph, then $Z(H)=I(H)$.

Proof. In a 2-hypergraph, the condition that a vertex $v$ can apply a force is equivalent to the condition that a subset of vertices (which in this case is a singleton) can infect an edge.

Let $H$ is a hypergraph and $W$ a subset of the vertices of $H$. The set of all vertices in $H$ that are infected after repeatedly applying the infection rule, with $W$ being the set of initially infected vertices, is called the derived set of $W$. This is denoted by $I_{W}$. A set $W$ is an infection set if and only if the derived set is the set of all vertices.

The empty hypergraph is the hypergraph with no vertices and no edges, we will not consider this case. A trivial hypergraph is hypergraph with vertices, but no edges. The infection number for any trivial hypergraph is clearly the number of vertices in the hypergraph since no set can ever infect any edge. For every other hypergraph, there is an upper bound on the size of the infection number for a hypergraph.

Proposition 1.2. Let $H$ be a non-trivial hypergraph on $n$ vertices and let $k$ be the size of the largest edge in $H$, then

$$
I(H) \leq n-k+1 .
$$

Proof. Let $A$ be a $(k-1)$-subset of an edge $E$ of size $k$ in the hypergraph. We claim that the set of all vertices except the vertices in $A$, form an infection set of size $n-(k-1)$ for the hypergraph. This follows since the final element in $E$ can infect $E$, since it cannot be adjacent to any uninfected vertices outside of $E$, as there are no uninfected vertices outside of $A$.

In Section 3 we will see that this bound holds with equality for the complete hypergraph; we will also demonstrate other hypergraphs where this bound is tight.

The line graph of a hypergraph $H$ is the graph formed by representing each edge of $H$ by a vertex; these vertices are adjacent in the line graph if and only if the corresponding edges of the hypergraph $H$ intersect. The line graph of a hypergraph $H$ is denoted by $L(H)$, we consider these graphs in more detail in Section 7 A hypergraph is connected if and only if its line graph is connected, that is a hypergraph is connected if there is a path between any two vertices in the line graph. A connected component of a hypergraph is a maximal connected sub-hypergraph. It is not hard to see that the infection number of a hypergraph is the sum of the infection numbers of the connected components of the hypergraph.

Proposition 1.3. If $H$ is a hypergraph with connected components $H_{1}, \ldots, H_{t}$ then

$$
I(H)=\sum_{i=1}^{t} I\left(H_{i}\right) .
$$




\section{Reduced hypergraphs}

One significant difference between graphs and hypergraphs is that there is no restriction on the size of an edge in a hypergraph, where in a graph all edges have size two. This can lead to the situation where one edge of a hypergraph is a subset of another edge (a hypergraph that does not have this property is called reduced). The most extreme case of this is when the entire set of vertices forms an edge.

Proposition 2.1. Let $H$ be a hypergraph with vertex set $V$. If $V$ is an edge of $H$, then $I(H)=1$,

Proof. This follows simply from the fact that any one vertex can infect the edge containing all the vertices.

Consequently, the infection number of a hypergraph is not monotone under sub-hypergraphs; indeed, simply adding an edge that contains the entire vertex set of the hypergraph will reduce the infection number to one.

The next result shows that we can remove any edge that is a subset of another in a hypergraph without changing the infection number.

Proposition 2.2. Let $H$ be a hypergraph and assume that $E_{1}$ and $E_{2}$ are edges of $H$ with $E_{1} \subset E_{2}$, then $I(H)=I\left(H \backslash E_{1}\right)$.

Proof. Assume that $A$ is an infection set for $H$. If $A^{\prime} \subseteq A$ can infect an edge $E$ (with $E_{2} \not E E$ ), then $A^{\prime}$ can also infect $E$ in $H \backslash E_{1}$. So starting with $A$, the infection process in $H \backslash E_{1}$ can progress as it does in $H$. At some point in the infection process for $H$, a set $A^{\prime}$ infects the edge $E_{1}$. This set $A^{\prime}$ can also infect $E_{2}$, since all of the vertices in $E_{2} \backslash E_{1}$ must already be infected. Thus $A$ forms an infection set for $H \backslash E_{1}$, and hence $I(H) \geq I\left(H \backslash E_{1}\right)$.

Conversely, assume that $A$ is an infection set for $H \backslash E_{1}$. Assume that $A^{\prime} \subseteq A$ can infect an edge $E$, then $A^{\prime}$ is not adjacent to any uninfected vertices in $E_{2} \backslash E$, and thus $A^{\prime}$ is also not adjacent to any uninfected vertices in $E_{1} \backslash E$. So $A^{\prime}$ can infect $E$ in the hypergraph $H$, and the infection process in $H$, starting with $A$ can progress as it does in $H \backslash E_{1}$. Further, $E_{2}$ is infected at some point in the infection process in $H \backslash E_{1}$, at this step in $H$, the edge $E_{1}$ is infected. Thus $A$ is also an infection set for $H$ and hence $I(H) \leq I\left(H \backslash E_{1}\right)$. Thus $I(H)=I\left(H \backslash E_{1}\right)$.

This proof implies not only that $I(H)=I\left(H \backslash E_{1}\right)$, but also that an infection set for one of the hypergraphs is also an infection set for the other.

Many of the notations and concepts for graphs can be generalized to hypergraphs. For a hypergraph $H$, the vertex set is denoted by $V(H)$ and the edge set by $E(H)$. If $v$ is a vertex in a hypergraph $H$, then the degree of $v$, denoted $\operatorname{deg}(v)$, is the number of edges that contain $v$. Further, if $A \subseteq V(H)$, then the degree of the set $A$ is defined by

$$
\operatorname{deg}(A)=|\{E \in E(H): A \subseteq E\}| .
$$

Similar to the notation for standard graphs, we will use $\delta(H)$ to denote the minimum degree of a vertex in a hypergraph $H$. Any subset that has degree 1 can infect the edge that contains it. But Proposition 2.1 shows that a vertex of high degree can still infect an edge. 
A hypergraph is said to be reduced if no edge is the subset of another edge. A hypergraph can be reduced by simply removing all edges that are contained in another edge. If a hypergraph is not reduced, then it is possible that the infection number is equal to one, while the minimum degree is very large. This is not the case for reduced hypergraphs.

Lemma 2.3. If $H$ is a reduced hypergraph and $I(H)=1$, then $\delta(H)=1$.

Proof. Assume that $H$ is a reduced hypergraph with $I(H)=1$. Let $\{v\}$ be an infection set of size one and let $E$ be the first edge that $v$ infects. Then $v$ cannot be adjacent to any vertex outside of $E$. Since the hypergraph is reduced, this implies that $v$ is in no other edges and hence has degree one.

This lemma also implies that in a reduced hypergraph with $I(H)=1$, the vertex that does the first infection must have degree one. It is not hard to see that the minimum degree in a graph is a lower bound for the zero forcing number. It is not true in general that $\delta(H)$ is a lower bound for $I(H)$ for a hypergraph $H$. Lemma 3.1 in the next section shows that the complete hypergraph is such an example where the minimum degree of a hypergraph is much larger than the infection number.

Throughout the remainder of this paper, we will assume that our hypergraphs are reduced.

\section{Complete hypergraphs and complete bipartite hypergraphs}

Let $H$ be a non-empty $k$-hypergraph on $n$ vertices. Then, from Proposition 1.2 , the infection number of $H$ is no more than $n-k+1$. In this section, we consider some $k$-hypergraphs on $n$ vertices with infection number $n-k+1$.

The complete $k$-uniform hypergraph, denoted by $H_{n}^{(k)}$, has all $k$-subsets of $\{1, \ldots, n\}$ as its edges.

Lemma 3.1. For any $k$ and $n$ with $1 \leq k \leq n$, the value of $I\left(H_{n}^{(k)}\right)$ is $n-k+1$.

Proof. From Proposition 1.2 it follows that the infection number is no more than $n-k+1$. In fact, for this graph any set of $n-k+1$ vertices is an infection set.

To see that the infection number cannot be any smaller, assume that there are initially $k$ uninfected vertices in $H_{n}^{(k)}$, say $v_{1}, \ldots, v_{k}$. Let $A$ be any subset of the $n-k$ infected vertices that can infect some edge $E$. Then $E=A \cup B$ where $B$ is a proper subset of $\left\{v_{1}, \ldots, v_{k}\right\}$. We can assume that $v_{1} \in B$ and $v_{k} \notin B$. Since the hypergraph is the complete hypergraph, the edge $A \cup\left(B \backslash\left\{v_{1}\right\} \cup\left\{v_{k}\right\}\right)$ is an edge in the hypergraph. This means that $v_{k}$ is an uninfected vertex that is adjacent to $A$ outside of $E$. This is a contradiction, so no set of fewer than $n-k+1$ vertices can infect an edge.

The complete hypergraph $H_{n}^{(2)}$ is isomorphic to the complete graph $K_{n}$. The previous result shows that $I\left(H_{n}^{(2)}\right)=Z\left(K_{n}\right)=n-1$. In a graph $G$, a set of vertices in which any two are adjacent is called a clique; the complete graph is 
a clique. The size of the maximum clique in $G$ is usually denoted by $\omega(G)$ and the value of the zero forcing number is bounded below by $\omega(G)-1[6]$.

This is another point where a major difference between the zero forcing number and the infection number is apparent. There is no comparable bound for a hypergraph that contains a subgraph isomorphic to the complete hypergraph $H_{n}^{(k)}$. For example, adding the edge $\{1, \ldots, n\}$ to the complete hypergraph $H_{n}^{(k)}$ produces a hypergraph that contains $H_{n}^{(k)}$ as a sub-hypergraph, but has infection number 1 . Next we will see how to construct a $k$-hypergraph that has $H_{n}^{(k)}$ as a sub-hypergraph, and infection number equal to 1 .

Proposition 3.2. Let $H$ be any $k$-hypergraph. There exists a $k$-hypergraph $H^{\prime}$ such that $E(H) \subset E\left(H^{\prime}\right)$ and $I\left(H^{\prime}\right)=1$.

Proof. Assume that the vertex set for $H$ is $\{1, \ldots, n\}$. The construction of the hypergraph $H^{\prime}$ proceeds as follows. First, $\{1, \ldots, n\}$ will be vertices in $H^{\prime}$, and all the edges of $H$ will be edges of $H^{\prime}$.

Second, the vertices of $H$ can be covered by $r_{1}=\left\lceil\frac{n}{k-1}\right\rceil$ sets each of size $k-1$. Call these sets $A_{1}, A_{2}, \ldots A_{r_{1}}$. Add the vertices $\left\{n+1, \ldots, n+r_{1}\right\}$ to the vertex set of $H$ and add sets

$$
A_{1} \cup\{n+1\}, A_{2} \cup\{n+2\}, \ldots, A_{r_{1}} \cup\left\{n+r_{1}\right\}
$$

to the edge set of $H^{\prime}$.

Third, the vertices $\left\{n+1, n+2, \ldots, n+r_{1}\right\}$ can be covered in $r_{2}=\left\lceil\frac{r_{1}}{k-1}\right\rceil$ sets each of size $k-1$. Call these sets $B_{1}, B_{2}, \ldots, B_{r_{2}}$, and add the sets

$$
B_{1} \cup\left\{n+r_{1}+1\right\}, B_{2} \cup\left\{n+r_{1}+2\right\}, \ldots, B_{r_{2}} \cup\left\{n+r_{1}+r_{2}\right\}
$$

to the edge set of $H^{\prime}$, and include the additional vertices $\left\{n+r_{1}+1, \ldots, n+\right.$ $\left.r_{1}+r_{2}\right\}$ in $H^{\prime}$.

Continue in this fashion until $r_{j}=1$. The final vertex to be added to the hypergraph $H^{\prime}$ is $n+r_{1}+r_{2}+\cdots+r_{j}$. This final vertex is an infection set for the $k$-hypergraph $H^{\prime}$.

Not only are there hypergraphs with infection number 1 that contain a complete hypergraph, but there are also $k$-hypergraphs on $n$ vertices with infection number $n-k+1$ which are not complete hypergraphs.

Proposition 3.3. For $2 \leq k \leq n$, let $H$ be the $k$-hypergraph on $n$ vertices that is formed by adding the element $n$ to each of the edges in $H_{n-1}^{(k-1)}$. If $n \geq 2 k-1$, then the infection number of $H$ is $n-k$; otherwise, it is $n-k+1$.

Proof. First consider the case where $n \geq 2 k-1$. Assume that the vertices labeled $1, \ldots, n-k$ are infected and the vertices labeled $n-k+1, \ldots, n$ are not infected. Thus there are $k$ uninfected vertices including $n$. Since $n \geq 2 k-1$ there are at least $k-1$ infected vertices, and any $(k-1)$-set of infected vertices can infect the vertex $n$. Once $n$ is infected, there are only $k-1$ uninfected vertices remaining. By construction, they will be in an edge with $n$ and thus can all be infected by $n$. Thus $I(H) \leq n-k$.

To see that the infection number cannot be less than $n-k$, let $A$ be any set of infected vertices of size $n-k-1$ and assume that the remaining $k+1$ vertices are uninfected. Thus there must be at least $k$ vertices from $\{1, \ldots, n-1\}$ that 
are uninfected. An argument similar to that used in the proof of Lemma 3.1 will show that $A$ cannot be an infection set.

Next consider the case where $n \leq 2 k-2$. Since $n-k+1$ is always an upper bound on the infection number, we only need to prove that no set of $n-k$ vertices can be an infection set. If $n=k$ this is trivial, so assume that $n>k$. Let $A$ be a set of $n-k$ infected vertices and assume the remaining $k$ vertices are uninfected. Since $|A| \leq k-2$, for every edge containing an uninfected vertex $v \in\{1, \ldots, n-1\}$ and a subset $A^{\prime}$ of $A$, there will be at least one uninfected vertex $w \in\{1, \ldots, n-1\}$ not in the edge. By construction of $H^{\prime}$, there will be another edge containing $A^{\prime}$ and $w$, and thus the set $A$ can never infect an edge.

The zero forcing number of the complete bipartite graph $K_{n_{1}, n_{2}}$ is known to be $n_{1}+n_{2}-2$. We derive a similar result for complete $k$-partite hypergraphs. Define the $k$-partite complete hypergraph $H_{n_{1}, n_{2}, \ldots, n_{k}}^{(k)}$ as follows. The vertex set of $H_{n_{1}, n_{2} \ldots, n_{k}}^{(k)}$ is $V$, which can be partitioned into $k$ disjoint parts, namely $V_{1}, V_{2}, \ldots, V_{k}$, where $\left|V_{i}\right|=n_{i}$. The edge set is the set of all $k$-sets with exactly one element from each of $V_{i}$ where $i=1, \ldots, k$.

Lemma 3.4. If $H=H_{n_{1}, n_{2}, \ldots, n_{k}}^{(k)}$ is a k-partite complete hypergraph, then $I(H)=n_{1}+n_{2}+\cdots+n_{k}-k$.

Proof. If we choose one vertex in each of $V_{i}$ for $i=1, \ldots, k$ to be uninfected and the remaining vertices to be infected, then we have an infection set of the appropriate size.

Similar to the case for the complete hypergraph (see the proof of Lemma 3.1), if there are two uninfected vertices in some $V_{i}$, then no set can infect either of these vertices.

The complete bipartite graph $K_{1, n}$ is also called a flower or a star. For this graph, the intersection of any two edges is the fixed single vertex in the first partition. This structure can be generalized to a hypergraph. If $H=$ $\left\{E_{1}, E_{2}, \ldots, E_{n}\right\}$ is a hypergraph with the property that for any two distinct $i, j \in\{1, \ldots, n\}$ the intersection $E_{i} \cap E_{j}=A$ (where $A$ is a non-empty set), then $H$ is called a flower. The sets $E_{i}$ are called the petals.

Lemma 3.5. Let $H$ be a hypergraph that is a flower with $p$ petals. Then $I(H)=$ $p-1$.

Proof. An infection set can be formed by taking one vertex of degree 1 from all but one of the petals. Each of these vertices can infect the edge that contains it. Then any vertex in the intersection of all the edges can infect the final edge.

\section{Interval hypergraphs and cyclic interval hy- pergraphs}

It is well-known for a graph $G$ that $Z(G)=1$ if and only if $G$ is a path, and if $G$ is a cycle, then $Z(G)=2$ (see [6]). In this section we will consider hypergraphs that are analogous to paths and cycles. 
Paths and cycles both have the property that the degree of every vertex is no more than 2. For hypergraphs there is an analogous bound on the infection number.

Lemma 4.1. Let $H$ be a connected $k$-hypergraph in which every vertex has degree no more than 2 , then $I(H) \leq k$.

Proof. We claim that any edge of $H$ is an infection set. Let $E=\left\{v_{1}, \ldots, v_{k}\right\}$ be any edge in $H$, and assume that $v_{1}, \ldots, v_{k}$ are all initially infected. Each $v_{i}$ is contained in only one edge, other than $E$, and hence can infect that edge. The same now holds for all other vertices in the newly infected edges. Since $H$ is connected, all vertices will be infected under this process.

Another way to generalize paths to hypergraphs is by linear hypergraphs. A hypergraph $H$ is linear if $\left|E_{i} \cap E_{j}\right| \leq 1$ for any two edges $E_{i}$ and $E_{j}$ of $H$. This implies that any set of two of more vertices can be in at most one edge, so a set of more than one vertex will have degree at most one.

Lemma 4.2. If $H$ is a reduced connected, linear hypergraph and all the vertices have degree no more than two, then $I(H) \leq 2$. Further, $I(H)=1$ if and only if $H$ has a vertex of degree one.

Proof. Let $H$ be a connected, linear hypergraph in which all vertices have degree no more than 2. Since $H$ is linear, any two adjacent vertices are contained in exactly one edge; thus any two adjacent vertices can infect the edge that contains them. Since $H$ is connected, starting with any two adjacent vertices being initially infected, the infection process will infect every vertex in the hypergraph.

If $H$ has a vertex with degree one, then this vertex can infect the edge that contains it. Then the vertices in this edge are each in at most one other edge, which they can now infect. Continuing like this infects all the vertices of the hypergraph. The converse holds by Lemma 2.3.

Next we consider a different generalization of paths in which the degrees of the vertices can be more than two. An interval ordering of the vertices of a hypergraph is an ordering of the vertices so that every hyperedge of the hypergraph is an interval of the ordering. We say that a hypergraph is an interval hypergraph if there exists an interval ordering of the vertices of the hypergraph.

Lemma 4.3. If $H$ is a reduced interval hypergraph, then $I(H)$ is equal to the number of connected components.

Proof. Since $H$ is an interval hypergraph, there is a linear ordering of the vertices and this ordering extends to the edges $\left(E_{1}<E_{2}\right.$ if this minimal element of $E_{1}$ is smaller than the minimal element of $\left.E_{2}\right)$.

Let $v_{1}$ be the first vertex in the ordering, and suppose $v_{1} \in E$. Since $H$ is reduced, it follows that $v_{1}$ has degree one, and is not adjacent to any other vertices outside of $E$. Thus $v_{1}$ can infect $E$. Let $v_{2}$ be the least vertex in the ordering that is not infected. Similar to $v_{1}$, this vertex can infect the largest edge that contains it. Continuing like this will eventually infect all the vertices in the connected component that contains $v$. The result then follows from Proposition 1.3. 
To generalize a cycle, we define a hypercycle to be a set of edges $E_{1}, E_{2}, \ldots, E_{k}$ with $E_{i} \cap E_{j} \neq \emptyset$ if and only if $|i-j| \equiv 1(\bmod k)$. In a hypercycle the maximum degree of a vertex is two.

Proposition 4.4. Let $H$ be a hypercycle, then $I(H) \leq 2$. Further, $I(H)=1$ if and only if $H$ has a vertex of degree 1.

Proof. If $H$ does not have a vertex of degree one, then pick one vertex in $E_{1} \cap E_{2}$ and one vertex in $E_{2} \cap E_{3}$. These two vertices can infect the edge $E_{2}$. Then the vertices in $E_{2} \cap E_{3}$ can infect $E_{3}$. Continuing like will infect all the remaining vertices in the hypergraph. By Lemma 2.3, $I(H)=2$, unless $H$ has vertex of degree one.

If $v \in E_{i}$ is a degree one vertex, then $v$ can infect $E_{i}$. Then the remaining vertices in $H$ can be infected as before, so $I(H)=1$.

The vertices of a hypergraph have an arc-ordering if the vertices can be cyclically ordered so that every edge is an arc, that is a set of vertices that are consecutive in this ordering. A hypergraph is a circular-arc hypergraph if there exists an arc-ordering of the vertices in the hypergraph.

One example of a circular-arc hypergraph is what we call the $t$-tight $k$ uniform cycle on $n$-vertices. This hypergraph is denoted by $C_{n}^{(k)}(t)$, and for it to be well-defined, we must assume that $k-t$ divides $n$. The vertex set of $C_{n}^{(k)}(t)$ is $\{1, \ldots, n\}$ and the following is the hyperedge set:

$$
\begin{aligned}
E_{1} & =\{1,2, \ldots, k\} \\
E_{k-t+1} & =\{k-t+1, k-t+2, \ldots, 2 k-t\}, \\
E_{2 k-2 t+1} & =\{2 k-2 t+1,2 k-2 t+2, \ldots, 3 k-2 t\}, \\
\vdots & \\
E_{n-k+t} & =\{n-k+t, \ldots, t-1, t\}
\end{aligned}
$$

(entries taken modulo $n$ ). For $i=0, \ldots, \frac{n}{k-t}-1$, the edge $E_{i(k-t)+1}$ is an arc that starts with the vertex $i(k-t)+1$. The function $f_{i}: V\left(C_{n}^{(k)}(t)\right) \rightarrow V\left(C_{n}^{(k)}(t)\right)$ defined by $f_{i}(v)=v+i(k-t)$ (modulo $n$ ) is an automorphism of the hypergraph that maps edge $E_{j}$ to $E_{i(k-t)+j}$. So $C_{n}^{(k)}(t)$ is edge transitive.

We consider the special case when $t=k-1$; this is the largest possible value of $t$, and $k-t=1$, so such a hypergraph is well-defined for all values of $k$ and $n$. We start with a simple lemma about the structure of this graph.

Lemma 4.5. If $k+1 \leq n<2 k-1$, then any pair of vertices from $C_{n}^{(k)}(k-1)$ is contained in at least two edges.

Proof. Let $x$ and $y$ be two vertices with $x<y$. If $y-x \leq n-k$, since $n-k \leq k-1$, then $x \notin E_{y}$, but both $x$ and $y$ will be in the edges $E_{y-k+1}, E_{y-k+2}, \ldots, E_{x}$ (subscripts taken modulo $n$ ). Conversely, if $y-x>n-k$, then both $x$ and $y$ are in the edges $E_{x-k+1}, \ldots, E_{y}$. This shows that for these values of $n$ and $k$ any pair of vertices will be in at least two edges.

Next we give the exact value of the infection number for $C_{n}^{(k)}(k-1)$. 
Lemma 4.6. Let $H=C_{n}^{(k)}(k-1)$.

1. If $n \geq 2 k-1$, then $I(H)=2$.

2. If $k+1 \leq n<2 k-1$, then $I(H)=\min \{i+1, n-k+1\}$ where

$$
i=\left\lceil\frac{k-1}{n-k}\right\rceil \text {. }
$$

3. If $n=k$, then $I(H)=1$.

Proof. Statement (3) is trivial, since if $n=k$, the hypergraph has only one edge. So we consider Statements (1) and (2).

Observe that once one edge is infected, the remainder of the hypergraph can be infected. For example, if $E_{1}$ is infected, then the set $\{2, \ldots, k\}$ will infect $E_{2}$. Subsequently, $\{3, \ldots, k+1\}$ will infect $E_{3}$ and so on.

If $n \geq 2 k-1$, then the set $A=\{1, k\}$ is a subset of $E_{1}$ and no other edge. Thus if $A$ is initially infected, it can infect the remaining vertices in $E_{1}$. Finally, since no vertex is contained in only one edge, the infection number cannot be one. This proves statement (1).

Assume that $k+1 \leq n<2 k-1$. To prove statement (2), we first show that infection sets of size $i+1$ and $n-k+1$ are possible for all $n$ and $k$.

As shown in Proposition 1.2 an infection set of size $n-k+1$ can be constructed for any non-empty hypergraph. To see that an infection set of size $i+1$ can be constructed for $H=C_{n}^{(k)}(k-1)$, let

$$
i=\left\lceil\frac{k-1}{n-k}\right\rceil
$$

and consider the subset

$$
A=\{1, n-k+1,2(n-k)+1, \ldots,(i-1)(n-k)+1, k\} \subset E_{1} .
$$

If $v_{1}$ and $v_{2}$ are vertices in $A$ such that $v_{2}$ follows $v_{1}$ in the order shown, then $v_{2}-v_{1} \leq n-k$ and so $v_{1} \notin E_{v_{2}}$. Thus $A$ only occurs as a subset of $E_{1}$ and so can infect $E_{1}$.

We now show by contradiction that an infection set of size less than $\min \{i+$ $1, n-k+1\}$ is not possible. Assume that $B$ is an infection set of size less than $\min \{i+1, n-k+1\}$. Then $|B| \leq i$ and $|B| \leq n-k$ and there are at least $k$ uninfected vertices. Since $H$ is edge transitive, we can assume that $B$ will first infect the edge $E_{1}$. Let $B^{\prime}=B \cap E_{1}$. Since $B$ infects $E_{1}$, there will be no uninfected vertices outside of $E_{1}$ that are adjacent to $B^{\prime}$.

Suppose $B^{\prime}$ contains only one element, $x$. Then there is at least one uninfected vertex not in $E_{1}$ and, since $n<2 k-1$, by Lemma 4.5 it will be in an edge with $x$ other than $E_{1}$. This is a contradiction, so $B^{\prime}$ must contain more than one vertex.

Let $B^{\prime}=\left\{v_{1}, \ldots, v_{\ell}\right\}$ where $v_{1}<v_{2}<\cdots<v_{\ell}$ and $2 \leq \ell \leq \min \{i, n-k\}$. If $v_{(j+1)}-v_{j}>n-k$ for any $j \in\{1, \ldots, \ell-1\}$, then $v_{j} \in E_{v_{(j+1)}}$ from which it follows that $B^{\prime} \subseteq E_{v_{(j+1)}}$. There are at least two uninfected vertices that are not in $E_{1}$. These vertices will be in $E_{v_{(j+1)}}$ and thus will be adjacent to $B^{\prime}$. This contradicts the assumption that $B^{\prime}$ infects $E_{1}$. Thus we must have $v_{(j+1)}-v_{j} \leq n-k$ for all $j \in\{1, \ldots, \ell-1\}$. 
Let $C=\left\{v_{1}, v_{1}+1, v_{1}+2, \ldots, v_{\ell}\right\}$. In other words, $C$ is the interval of length $v_{\ell}-v_{1}+1$ starting with $v_{1}$. Note that $B^{\prime} \subseteq C$. The vertices in the edges either starting with $v_{1}$ or ending with $v_{\ell}$ are all adjacent to $C$. If $n$ is sufficiently small, these are all vertices in the hypergraph (outside of $C$ ), otherwise they are a set of vertices of size $2\left(k-\left(v_{\ell}-v_{1}+1\right)\right)$. Thus the number of vertices adjacent to $C$ (but not in $C$ ) is equal to

$$
\min \left\{n-\left(v_{\ell}-v_{1}+1\right), 2\left(k-\left(v_{\ell}-v_{1}+1\right)\right)\right\} .
$$

Since $k-\left(v_{\ell}-v_{1}+1\right)$ of these vertices are in $E_{1}$, the number of vertices not in $E_{1}$ that are adjacent to $C$ is

$$
\min \left\{n-k, k-\left(v_{\ell}-v_{1}+1\right)\right\} .
$$

The number of infected vertices adjacent to $C$ and not in $E_{1}$ is at most $|B|-\ell$. We now show that this number is less than the total number of vertices adjacent to $C$ and not in $E_{1}$. This proves that there is an uninfected vertex, not in $E_{1}$, that is adjacent to $B^{\prime}$, which is a contradiction.

Suppose that the number of vertices adjacent to $C$ and not in $E_{1}$ is $n-k$ (i.e. $\left.n-k \leq k-\left(v_{\ell}-v_{1}+1\right)\right)$. Since $|B| \leq n-k$ and $\ell \geq 2$, it follows that $|B|-\ell<n-k$ and so there is an uninfected vertex outside of $E_{1}$ that is adjacent to $B^{\prime}$.

Now suppose that $k-\left(v_{\ell}-v_{1}+1\right)<n-k$. It follows from $v_{(j+1)}-v_{j} \leq n-k$ for all $j \in\{1, \ldots, \ell-1\}$ that $v_{\ell}-v_{1} \leq(\ell-1)(n-k)$. Therefore,

$k-\left(v_{\ell}-v_{1}+1\right) \geq k-1-(\ell-1)(n-k)=\left(\frac{k-1}{n-k}\right)(n-k)-(\ell-1)(n-k)$.

By definition $i-1<\frac{k-1}{n-k}$,

$$
k-\left(v_{\ell}-v_{1}+1\right)>(i-\ell)(n-k) \geq(|B|-\ell)(n-k) \geq|B|-\ell
$$

and again there is an uninfected vertex not in $E_{1}$ that is adjacent to $B^{\prime}$.

We consider one other special case, namely when $n=k+1$; this is the smallest non-trivial value for $n$. In this case, the number of edges is $\frac{n}{k-t}$, and each edge misses exactly one element.

Proposition 4.7. Let $t, k$ and $n$ be integers and assume that $k-t$ divides $k+1$. The infection number of $C_{k+1}^{k}(t)$ is equal to $\frac{k+1}{k-t}-1$.

Proof. To construct an infection set of this size take the vertex missing from every edge except one (call this edge $E$ ). These vertices only occur together in the edge $E$, so they can infect $E$. This leaves only one uninfected vertex (the vertex missing from $E$ ) which can be infected by any of the vertices in $E$.

Let $A$ be a set of size $\frac{k+1}{k-t}-2$. Each of the $\frac{k+1}{k-t}$ edges does not include a single vertex. Thus are there at least two edges that miss the vertices that are not contained in the set $A$. So $A$ must be a subset of both of these edges, and hence cannot infect them. 


\section{$5 \quad$ Hypergraphs from $t$-designs}

For a hypergraph $H$ there must be a set of size no more than $I(H)$ that causes the first infection. For this to be possible, this set must be contained in exactly one edge. Conversely, if every subset of vertices of size $t$ is contained in at least two edges, we can conclude that the infection number is strictly larger than $t$. This observation is useful in determining the infection number of hypergraphs that are themselves combinatorial designs.

A 2 - $(n, k, 1)$ design is a collection of $k$-sets (called blocks) from the base set $\{1, \ldots, n\}$ such that each pair from the base set occurs in exactly one $k$-set. The blocks of any $2-(n, k, 1)$ design form a linear hypergraph with $\frac{n(n-1)}{k(k-1)}$ edges. If a 2 - $(n, k, 1)$ design has $n=k^{2}-k+1$, then the design is a symmetric design; in this case any two blocks intersect. Further, given a $2-(n, k, 1)$ design, a subdesign is simply a collection of blocks from the design that forms a $2-(m, k, 1)$ design for some $m<n$. For more on designs see [4], or any standard reference on design theory.

Proposition 5.1. Let $H$ be a hypergraph in which the edges form a symmetric design with parameters $2-\left(k^{2}-k+1, k, 1\right)$. If $k \geq 3$, then the infection number of $H$ is 3 .

Proof. Assume the vertices of $H$ are $\{1, \ldots, n\}$ (and $n=k^{2}-k+1$ ). Each pair of vertices is contained in exactly one edge of $H$; thus any pair can infect the edge that contains it. If $k>2$ and all the vertices in a single edge are infected, and no other vertices are infected, then the process stops. Thus the infection number of $H$ must be greater than 2 .

Assume, without loss of generality, that $E=(1,2, \ldots, k)$ is a block in $H$. We claim that the set $\{1,2, k+1\}$ (or any triple, not contained in a single block) can infect the hypergraph.

First the vertices 1,2 can infect the edge $E$. At this stage there are $k+1$ vertices that are infected. Then for any $i \in\{1, \ldots, k\}$ the pair $i, k+1$ can infect the edge that contains both $i$ and $k+1$; call this edge $E_{i}$. For distinct $i, j$ the intersection of $E_{i}$ and $E_{j}$ is exactly $k+1$ (since $H$ is linear). So at this stage $k(k-2)$ new vertices are infected. Since $k(k-2)+k+1=k^{2}-k+1=n$, all the vertices in $H$ are infected.

The natural generalization of 2 - $(n, k, 1)$ designs are $t$ - $(n, k, 1)$ designs. A $t$ - $(n, k, 1)$ design is a set of $k$-subsets (called blocks) from $\{1, \ldots, n\}$ with the property that every $t$-subset occurs in exactly one of the $k$-subsets. We will consider hypergraphs in which the edge set forms a $t-(n, k, 1)$ design. Note that any $t$-set, will occur in a single block, and any $(t-i)$-set will occur in exactly $\left(\begin{array}{c}n-(t-i) \\ i\end{array}\right) /\left(\begin{array}{c}k-(t-i) \\ i\end{array}\right)$ blocks. Just as for 2-designs, the infection number for a hypergraph whose edges form a $t$ - $(n, k, 1)$ design must be at least $t$.

In this case, we need to consider sub-hypergraphs. If $H$ is a hypergraph and $W$ is a subset of vertices in $H$, then the sub-hypergraph induced by $W$ is the hypergraph with vertex set $W$ and the edges are exactly the edges from $H$ which only contain vertices from $W$.

Theorem 5.2. Let $H$ be a hypergraph in which the edges form a $t$ - $(n, k, 1)$ design. For a set $W$ of vertices from $H$, let $I_{W}$ denote the derived set of $W$. The sub-hypergraph of $H$ induced by $I_{W}$ is either a trivial hypergraph, or a $t$ - $\left(\left|I_{W}\right|, k, 1\right)$ design. 
Proof. If $|W|<t$, then $W$ cannot infect any edges of $H$. So the sub-hypergraph of $H$ induced by $I_{W}$ is empty.

If $|W|=t$, then $W$ can infect only the edge that contains it. This single edge is, trivially, a $t-(k, k, 1)$ design.

Let $H^{\prime}$ be the sub-hypergraph of $H$ induced by $I_{W}$. Any $t$-subset $S$ of $I_{W}$ is contained in a unique edge $E$ of $H$. Since all the vertices of $S$ are infected, the set $S$ can infect the edge $E$. This means that $E$ is an edge of $H^{\prime}$. So every $t$ subset of vertices from $I_{W}$ occur in an edge of $H^{\prime}$. Since $H^{\prime}$ is a sub-hypergraph of $H$, no $t$-subset can occur in more than one edge. Thus $H^{\prime}$ is a $t-\left(\left|I_{W}\right|, k, 1\right)$ design.

Corollary 5.3. Let $H$ be a hypergraph in which the edges form a $t-(v, k, 1)$ design. If the design does not contain any non-trivial sub-designs, then $I(H)=$ $t+1$.

Proof. Let $W$ be any set of $t+1$ vertices that are not contained in a single edge. Then the derived set $I_{W}$ is larger than $k$. So the sub-hypergraph of $H$ induced by $I_{W}$ is neither a trivial hypergraph, nor a single edge. Thus the induced subhypergraph is a non-trivial $t$ - $\left(\left|I_{W}\right|, k, 1\right)$ sub-design; since the $t-(v, k, 1)$ design has no non-trivial sub-design, it must include all edges of $H$. Thus $I_{W}$ includes all vertices of $H$ and $W$ is an infection set.

Let $q$ be a prime power. Consider the finite projective space $P G(n, q)$. The points of $P G(n, q)$ are the 1-dimensional subspaces of the vector space $\mathbb{F}_{q}^{n+1}$, the lines are the 2-dimensional subspaces of the vector space $\mathbb{F}_{q}^{n+1}$; incidence between points and lines is induced by incidence in the vector space [4, Section VI.7.5]. This space can be used to construct a $2-\left(\frac{q^{n+1}-1}{q-1}, q+1,1\right)$-design. For this we take the points of $P G(n, q)$ as points of the design, and the lines of $P G(n, q)$ as blocks.

Proposition 5.4. Let $H$ be the hypergraph with edge set equal to the blocks of a $2-\left(\frac{q^{n+1}-1}{q-1}, q+1,1\right)$-design constructed from the finite projective space $P G(n, q)$. Provided that $n \geq 2$, the infection number of $H$ is $n+1$.

Proof. First note that Theorem 5.2 implies that the infection number of $H$ must be at least $n+1$. The derived set of any set of vertices will be a subspace of the projective space, so if the derived set includes all the vertices $t$ must contain a spanning set. Finally, any spanning set will form an infection set.

\section{Hypergraph products}

In this section we will consider the behaviour of the infection number over various hypergraph products.

We begin with the direct product. For two hypergraphs $H_{1}$ and $H_{2}$ the direct product of $H_{1}$ and $H_{2}$ is the hypergraph with vertex set $V\left(H_{1}\right) \cup V\left(H_{2}\right)$ and edge set

$$
\left\{E_{1} \cup E_{2} \mid E_{1} \in E\left(H_{1}\right), E_{2} \in E\left(H_{2}\right)\right\} .
$$

The direct product of two hypergraphs is denoted by $H_{1} \times H_{2}$. The direct product can be defined recursively for any number of hypergraphs. Note that 
the size of the edges increases, so this is not an extension of the definition of the direct product of two graphs.

We have already seen an example of the hypergraph direct product. The $k$-partite complete graph $H_{n_{1}, n_{2}, \ldots, n_{k}}^{(k)}$ is isomorphic to the direct product of the $k$ complete graphs $H_{n_{i}}^{(1)}$ for $i=1, \ldots, k$. The case where $H_{1}$ is a complete graph and $H_{2}$ has only a single vertex was considered in Proposition 3.3. To start, we will generalize these results.

Theorem 6.1. Let $k_{i}$ and $n_{i}$ be integers for $i=1, \ldots, \ell$ with $k_{i} \leq n_{i}$. If $n_{i} \geq 2 k_{i}$ for at least one $i$, then

$$
I\left(H_{n_{1}}^{\left(k_{1}\right)} \times H_{n_{2}}^{\left(k_{2}\right)} \times \cdots \times H_{n_{\ell}}^{\left(k_{\ell}\right)}\right)=\sum_{i=1}^{\ell}\left(n_{i}-k_{i}\right) .
$$

Proof. Assume that $n_{1} \geq 2 k_{1}$, and we will construct an infection set $A$ of size $\sum_{i=1}^{\ell}\left(n_{i}-k_{i}\right)$. For each graph $H_{n_{i}}^{\left(k_{i}\right)}$ pick any $n_{i}-k_{i}$ vertices to be part of the set $A$; so $k_{i}$ of the vertices are uninfected in each sub-hypergraph. We show that $A$ is an infection set.

Let $E$ be an edge that contains $k_{1}$ of the infected vertices from $H_{n_{1}}^{\left(k_{1}\right)}$ (since $n_{1} \geq 2 k_{1}$ this is possible) and all of the $k_{i}$ uninfected vertices from the other sub-hypergraphs $H_{n_{i}}^{\left(k_{i}\right)}$ with $i=2, \ldots, \ell$. Then the $k_{1}$ infected vertices from $H_{n_{1}}^{\left(k_{1}\right)}$ never occur with an uninfected vertex outside of this edge, so they can infect this edge. Then the only uninfected vertices remaining in the hypergraph are the $k_{1}$ uninfected vertices from $H_{n_{1}}^{\left(k_{1}\right)}$. These vertices can be infected by any edge that contains them.

This is optimal since there cannot initially be more than $k_{i}$ uninfected vertices in any sub-hypergraph $H_{n_{i}}^{\left(k_{i}\right)}$. Thus

$$
I\left(H_{n_{1}}^{\left(k_{1}\right)} \times H_{n_{2}}^{\left(k_{2}\right)} \times \cdots \times H_{n_{\ell}}^{\left(k_{\ell}\right)}\right) \leq \sum_{i=1}^{\ell}\left(n_{i}-k_{i}\right) .
$$

Note that for the hypergraph in the previous theorem, $\sum n_{i}$ is the number of vertices and $\sum k_{i}$ is the size of the edges. So this theorem shows that the infection number of this product hypergraph is one less than the upper bound given in Proposition 1.2. If it is not the case that one of the sub-hypergraph $H_{n_{i}}^{\left(k_{i}\right)}$ has $n_{i} \geq 2 k_{i}$, then the infection number for the hypergraph is the upper bound.

Lemma 6.2. Let $k_{i}$ and $n_{i}$ be integers for $i=1, \ldots, \ell$ with $k_{i} \leq n_{i}$. If $n_{i}<2 k_{i}$ for all $i$, then

$$
I\left(H_{n_{1}}^{\left(k_{1}\right)} \times H_{n_{2}}^{\left(k_{2}\right)} \times \cdots \times H_{n_{\ell}}^{\left(k_{\ell}\right)}\right)=1+\sum_{i=1}^{\ell}\left(n_{i}-k_{i}\right) .
$$

Proof. By Proposition 1.2, there is an infection set of size

$$
n-k+1=1+\sum_{i=1}^{\ell}\left(n_{i}-k_{i}\right)
$$


To show that the infection set cannot be any smaller, assume that $A$ is an infection set of size $\sum_{i=1}^{\ell}\left(n_{i}-k_{i}\right)$. Initially, there cannot be more than $k_{i}$ uninfected vertices in any sub-hypergraph $H_{n_{i}}^{\left(k_{i}\right)}$. So we can assume that $A$ contains exactly $n_{i}-k_{i}$ vertices from each sub-hypergraph $H_{n_{i}}^{\left(k_{i}\right)}$. Since each $n_{i}<2 k_{i}$, no sub-hypergraph has an edge containing only infected vertices.

Let $A^{\prime} \subset A$ be the subset of infected vertices that does the first infection and assume it infects edge $E=E_{1} \cup \cdots \cup E_{\ell}$. For any $i$, the size of $A^{\prime} \cap E_{i}$ is no more than $n_{i}-k_{i}<k_{i}$, so there is an uninfected vertex in $H_{n_{i}}^{\left(k_{i}\right)}$. By construction this vertex is adjacent to $A^{\prime} \cap E_{i}$. This is a contradiction.

For any hypergraph $H$ the hypergraph $H \times H_{1}^{1}$ is the hypergraph formed by adding a single vertex to every edge of $H$. In some cases, this operation can reduce the infection number of the hypergraph. Consider the hypergraph $H$ on vertex set $\{1,2,3,4\}$ with edge set $\{\{1,2\},\{3,4\}\}$; this hypergraph has infection number two. Then $H \times H_{1}^{1}$ is the hypergraph on five vertices with edge set $\{\{1,2,5\},\{3,4,5\}\}$. The infection number of $H \times H_{1}^{1}$ is one. Our next result proves that the infection number cannot drop by more than one using this operation.

Theorem 6.3. For any hypergraph $H$

$$
I(H)-1 \leq I\left(H \times H_{1}^{1}\right) \leq I(H) .
$$

Moreover, $I\left(H \times H_{1}^{1}\right)=I(H)-1$ if and only if $H$ is disconnected and has at least one component which is a single edge.

Proof. By definition, an infection set of $H$ is also an infection set of $\left(H \times H_{1}^{1}\right)$. As a result, it follows that $I\left(H \times H_{1}^{1}\right) \leq I(H)$.

First we will show that if either $H$ is connected, or $H$ is disconnected with no component being a single edge, then

$$
I(H) \leq I\left(H \times H_{1}^{1}\right) .
$$

Let $v$ be the vertex that is added to every edge in $H$ to form $H \times H_{1}^{1}$.

If $H$ is a single edge $I\left(H \times H_{1}^{1}\right)$ and $I(H)$ both equal 1. We assume that $H$ is not a single edge. Further, let $A$ be an infection set of $H \times H_{1}^{1}$ with cardinality $I\left(H \times H_{1}^{1}\right)$. Since $H$ contains at least 2 edges, $A \neq\{v\}$. In fact, we will show that $A$ does not contain the vertex $v$.

To the contrary, suppose that $v \in A$. Let $A^{\prime} \subset A$ be the set that causes the first infection in the process; assume that it infects the edge $E$ of $H \times H_{1}^{1}$. By the infection rule, there is no uninfected vertex $u$ outside of $E$ such that $\{u\} \cup A^{\prime}$ is a subset of another edge. In fact, noting that every edge of $H \times H_{1}^{1}$ contains vertex $v$, we see that $\{u\} \cup\left(A^{\prime} \backslash\{v\}\right)$ is not contained in any edge other than $E$. Thus, $A^{\prime} \backslash\{v\}$ can infect $E$, and hence the vertex $v$. This implies that $A \backslash\{v\}$ is also an infection set of $H \times H_{1}^{1}$, which contradicts the choice of $A$ being a minimum infection set. Thus no minimum infection set of $H \times H_{1}^{1}$ contains $v$.

Let $H_{i}$, for $i=1, \ldots, c$, be the components of $H$ and assume that no component is a single edge (note that $c=1$ if and only if $H$ is connected). Consider the sub-hypergraph $H_{i} \times H_{1}^{1}$ in $H \times H_{1}^{1}$. Let $B_{i}$ be an infection set for $H_{i} \times H_{1}^{1}$ with minimal size. Since no $H_{i}$ is a single edge, no $B_{i}$ contains the vertex $v$. The infection set of $H \times H_{1}^{1}$ with the minimal cardinality must contain a 
subset $B_{i}$ for each $i=1, \ldots, c$. Thus $\bigcup_{i=1}^{c} B_{i}$ is an infection set of $H$, and $I(H) \leq I\left(H \times H_{1}^{1}\right)$.

Next suppose that $H_{i}$ for $i=1, \ldots, \ell$ are the components of $H$ that are a single edge. For each $H_{i}$, with $i>\ell$, let $B_{i}$ be an infection set of $H_{i} \times H_{1}^{1}$ with minimum cardinality; from above this infection set does not contain $v$.

The infection number of each $H_{i}$ with $i=1, \ldots, \ell$ is 1 , since each is a single edge. Further, $v$ can only infect one edge in $\left(H \times H_{1}^{1}\right)$, since it is contained in every edge of the hypergraph. This implies that an infection set for $H \times H_{1}^{1}$ must contain at least $\ell-1$ vertices, other than $v$, to infect the edges $H_{i} \times H_{1}^{1}$ for $i=1, \ldots, \ell$. For $i=1, \ldots, \ell$, we select a single vertex $v_{i}$ from $H_{i}$. Then $\left(\bigcup_{i=1}^{c} B_{i}\right) \cup\left(\bigcup_{i=1}^{\ell-1}\left\{v_{i}\right\}\right)$ is an infection set of $H \times H_{1}^{1}$ with minimal size. But, $\left(\bigcup_{i=1}^{n} B_{i}\right) \cup\left(\bigcup_{i=1}^{\ell-1}\left\{v_{i}\right\}\right)$ is an infection set of $H$. This implies that $I(H) \leq$ $I\left(H \times H_{1}^{1}\right)+1$.

Conversely, note that each infection set of $H$ with cardinality $I(H)$ contains exactly one vertex from each single edge. Thus $I(H)=\left|\left(\bigcup_{i=1}^{c} B_{i}\right) \cup\left(\bigcup_{i=1}^{\ell}\left\{v_{i}\right\}\right)\right|$, (where $B_{i}$ is the infection set of $H_{i}$ for $i>\ell$, and $v_{i}$ is any vertex in $H_{i}$ with $i=1, \ldots, \ell$ ). Exactly one of the vertices $v_{i}$ may be removed from this infection set for $H$ to form an infection set for $H \times H_{1}^{1}$ (since the $v$ can infect the edge $\left.H_{i}\right)$.

There is a simple bound on the infection number for the direct product of any two hypergraphs.

Lemma 6.4. Let $H_{1}$ and $H_{2}$ be two hypergraphs, then

$$
I\left(H_{1} \times H_{2}\right) \leq I\left(H_{1}\right)+I\left(H_{2}\right) .
$$

Proof. If $A$ is an infection set for $H_{1}$, and $B$ is an infection set for $H_{2}$, then $A \cup B$ is an infection set for $H_{1} \times H_{2}$.

To see this assume that $A_{1} \subset A$ infects the edge $E$, and $B_{1} \subset B$ infects the edge $F$. Then $A_{1} \cup B_{1}$ is a subset of infected vertices in $E \cup F$. We claim that $A_{1} \cup B_{1}$ can infect $E \cup F$. Assume that there is an uninfected vertex $v$, outside of $E \cup F$, that is adjacent to $A_{1} \cup B_{1}$. If $v$ is a vertex from $H_{1}$, then $A_{1} \cup\{v\}$ is contained an edge other than $E$. This contradicts the fact that $A_{1}$ infects $E$. Similarly, $v$ cannot be a vertex of $H_{2}$, thus no such vertex $v$ can exist.

The next example shows that this bound can hold with equality.

Proposition 6.5. If $H_{1}$ and $H_{2}$ are both hypergraphs with more than one edge and $I\left(H_{1}\right)=1$ and $I\left(H_{2}\right)=1$, then $I\left(H_{1} \times H_{2}\right)=2$.

Proof. Since both $H_{1}$ and $H_{2}$ have more than one edge, no vertex of $H_{1} \times H_{2}$ has degree one, so by Lemma 2.3. $I\left(H_{1} \times H_{2}\right)>1$. The previous lemma result then gives the result.

Next we define two additional constructions of hypergraphs that are based on the corona of a graph. The join of a graph $G$ with a vertex $v$, is the graph with vertex set $V(G) \cup\{v\}$, and the edge set is the set of all the edges from $G$ along with all edges of the form $\{v, h\}$ where $h \in V(G)$. So it is the graph formed by adding $v$ to $G$ and making every vertex in $G$ adjacent to $v$. If $G$ and $H$ are graphs, the $H$-corona of $G$ is the graph formed by taking $G$ and for each vertex in $G$ joining a copy of $H$ to the vertex. The $H$-corona of $G$ is denoted 
by $G \circ H$. In $[\underline{6}$ an upper bound on the zero forcing number for the corona of a graph is given; this bound follows from a simple construction of a zero forcing set for $G \circ H$ using zero forcing sets of $G$ and $H$.

Theorem 6.6. [6, Prop. 2.12] Let $G$ and $H$ be graphs, then

$$
Z(G \circ H) \leq Z(G)|V(H)|+(|V(G)|-Z(G)) Z(H) .
$$

This first generalization of a corona to hypergraphs we will call the weak corona. Let $H$ be a $(k-1)$-hypergraph. Define the join of $H$ with a vertex $\{v\}$ to be the hypergraph formed by adding $v$ to every edge of $H$. Define the weak $H$-corona of a $k$-hypergraph $G$ to be the $k$-hypergraph formed by taking $G$ and joining a copy of $H$ to each vertex of $G$. This new hypergraph is denoted by $G \circ_{w} H$. If $H$ is a hypergraph with $\ell$ disjoint edges, then the $H$-corona of a hypergraph $G$ is an example of an $\ell$-corona of $G$ as defined in [3]. Figure 1 is the weak corona of a cycle with a single edge on two vertices.

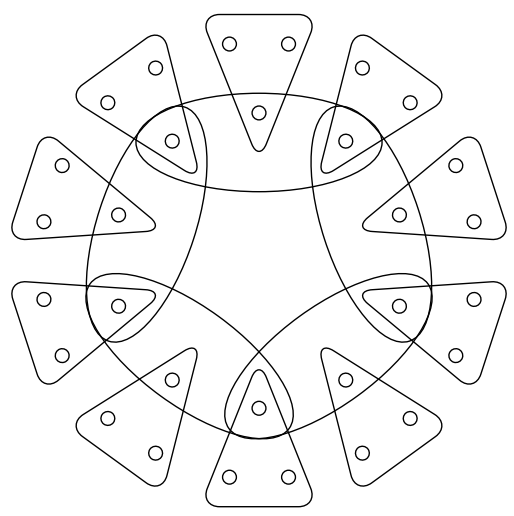

Figure 1: The weak corona of a cycle with an edge

Using a construction of an infection set based on the construction used in the proof of Theorem 6.6, we can establish a similar bound.

Theorem 6.7. Let $G$ and $H$ be hypergraphs, then

$$
I\left(G \circ{ }_{w} H\right) \leq|V(G)| I(H) .
$$

Proof. In $G \circ_{w} H$, there is a copy of $H$ for each vertex $g$ in $G$; call this subhypergraph $H_{g}$. The vertices of $G \circ_{w} H$ are either $g \in V(G)$, or $h_{g} \in V\left(H_{g}\right)$.

Let $A$ be an infection set for $H$ and let $A_{g}$ be the copy of $A$ in the hypergraph $H_{g}$. Define $B=\bigcup_{g \in G} A_{g}$. We claim that $B$ is an infection set for $G \circ_{w} H$.

If $A^{\prime} \subset A$ infects an edge $E$ in $H$, then $A_{g}^{\prime} \subset A_{g}$ (the set of vertices corresponding to $A^{\prime}$ in $H_{g}$ ) can infect the edge $E_{g}$ in $H_{g}$ corresponding to $E$. The edge $E_{g}$ contains $g$, so this vertex will also be infected. Thus following the infection process for $H$, the set $A_{g}$ infects all the vertices in $H_{g}$ (including $g$ ) for all the $g \in V(G)$. Therefore the set $A$ is an infection set for $G \circ_{w} H$.

We will consider another generalization of the corona to hypergraphs. Let $H$ be a $k$-hypergraph. Define the strong join of $H$ with a vertex $v$ to be the 
hypergraph with vertex set $V(H) \cup\{v\}$, and edge set containing all edges of $H$, together with all edges of the form $F \cup\{v\}$ where $F$ is a $(k-1)$-subset of an edge in $H$. Denote this graph by $H \nabla v$.

Let $H$ be a $k$-hypergraph. The strong $H$-corona of a $k$-hypergraph $G$ is defined to be the $k$-hypergraph formed by taking first a copy of $G$, and then for each vertex $g \in V(G)$ taking a disjoint copy of $H$ and $H \nabla g$. This hypergraph is denoted by $G \circ_{s} H$. The number of vertices in $G \circ_{s} H$ is $|V(G)|+|V(G)||V(H)|$ and the number of edges is

$$
|E(G)|+|V(G)||E(H)|(k+1) .
$$

Theorem 6.8. Let $G$ and $H$ be hypergraphs, then

$$
I\left(G \circ \circ_{w} H\right) \leq I(G)|V(H)|+(|V(G)|-I(G)) I(H) .
$$

Proof. For each vertex $g \in V(G)$, let $H_{g}$ denote the copy of $H$ corresponding to $g$.

Construct an infection set $A$ for $G \circ_{s} H$ as follows. Let $B$ be a minimal infection set for $G$. For every $g \in B$, set all the vertices in $H_{g}$, except $g$, to be in $A$ (so initially infected). For every vertex $g$ of $G$ not in $B$, add a minimal infection set from $H_{g}$ to $A$.

For every $g \in B$, the vertices of $H_{g}$ are all infected, so they can infect $g$. Then the vertices $g \in B$ from the copy of $G$ in $G \circ_{w} H$ can infect some vertices in $G$. Assume that $g_{1}$ is one of these vertices infected in the first step of the infection process for $B$. Then $g_{1}$ is initially uninfected, and $A$ includes an infection set from $H_{g_{1}}$. Once $g_{1}$ is infected, the infection set from $H_{g_{1}}$ can infect the vertices in $H_{g_{1}}$. Now $g_{1}$ can infect other vertices in the copy of $G$. Continuing like this, all of the vertices in $G \circ_{w} H$ will be infected.

The next product that we consider is the Cartesian product. If $H$ and $G$ are hypergraphs, $G \square H$ is the Cartesian product. The vertex set of this hypergraph is $V(G) \times V(H)$. For a set $E=\left\{e_{1}, e_{2}, \ldots, e_{k}\right\}$ and a vertex $v$, define

$$
E \times v=\left\{\left(e_{1}, v\right),\left(e_{2}, v\right), \ldots,\left(e_{k}, v\right)\right\}, \quad v \times E=\left\{\left(v, e_{1}\right),\left(v, e_{2}\right), \ldots,\left(v, e_{k}\right)\right\}
$$

The edge set of the Cartesian product is

$$
\{E \times v: E \in E(G), v \in V(H)\} \cup\{u \times E: u \in V(G), E \in E(H)\} .
$$

There is a well-known simple upper bound for the zero forcing number of the Cartesian product of two graphs (see [6]);

$$
Z(G \square H) \leq Z(G)|V(H)| .
$$

We generalize this result for the infection number of the Cartesian product to hypergraphs. For this we first introduce a generalization of the infection number. Consider the following rule.

$m$-Infection Rule: A set $A$, with $|A| \geq m$, of infected vertices can infect the vertices in an edge $E$ if:

1. $A \subset E$, and

2. there are no uninfected vertices $v$, not contained in $E$, such that $A \cup\{v\}$ is a subset of an edge. 
For $m=1$ this corresponds to our normal infection rule. A set of vertices of a hypergraph is an $m$-infection set when the vertices of the set are initially infected and applying the $m$-infection rule repeatedly infects all vertices of the hypergraph. The $m$-infection number of a hypergraph is the size of a smallest infection set for $H$. The $m$-infection number is denoted by $I_{m}(H)$. It is clear that $I_{m}(H) \leq I_{m+1}(H)$. Similarly, $I_{m+1}(H)$ can be bounded in terms of $I_{m}(H)$.

Lemma 6.9. Let $H$ be a hypergraph. Then

$$
I_{m+1}(H) \leq I_{m}+|E(H)| .
$$

Proof. Let $A$ be an $m$-infection set. Let $\mathcal{E}$ be the edges of $E(H)$, which are not completely contained in $A$. Let $f$ be a function from $\mathcal{E}$ to $V(H)$ that maps $E \in \mathcal{E}$ to one element of $E \backslash A$.

Let $B$ denote the set $A \cup\{f(E): E \in \mathcal{E}\}$. We claim that $B$ is an $(m+1)$ infection set. Clearly $B$ infects all the edges of $H$ that are not in $\mathcal{E}$. Since $A$ is an $m$-infection set, there is a set $A^{\prime} \subseteq A$ of size $m$ and an edge $E \in \mathcal{E}$ such that $A^{\prime}$ can $m$-infect $E$. Hence, $E$ is the only edge containing $A^{\prime} \cup\{f(E)\}$, so $A^{\prime} \cup\{f(E)\}$ can $(m+1)$-infect $E$. Continuing like this (following the $m$-infection process with $A), B$ is an $(m+1)$-infection set. As $|\mathcal{E}| \leq|E(H)|$, this shows the assertion.

This bound can be tight, for example if $H$ consists of one single edge of size at least 2 .

Theorem 6.10. Let $G$ and $H$ be hypergraphs. Then

$$
I(G \square H) \leq I(G) I_{2}(H) .
$$

Proof. Let $A_{G}$ be an infection set of $G$ and $A_{H}$ a 2-infection set of $H$. We define a set

$$
A=\left\{\left(a_{G}, a_{H}\right): a_{G} \in A_{G}, a_{H} \in A_{H}\right\},
$$

we will show that $A$ is an infection set of $G \square H$. To start, define

$$
A_{1}=\left\{\left(a_{G}, a_{H}\right): a_{G} \in A_{G}, a_{H} \in V(H)\right\} .
$$

We will show that $A$ infects all the vertices of $A_{1}$.

Let $E_{H}$ be an edge of $H$. In some step of the 2-infection process of $H$, the edge $E_{H}$ is 2-infected by a set $A_{H}^{\prime}$ (starting from $A_{H}$ ). We want to show that for a given $v \in A_{G}$, the edge $v \times E_{H}$ is the only edge of $G \square H$ that contains $\{v\} \times A_{H}^{\prime}$. Suppose that there is an edge $E$ of $G \square H$ that contains $\{v\} \times A_{H}^{\prime}$. The set $A_{H}^{\prime}$ has size at least 2 , so $E$ contains vertices $\left(v, w_{1}\right)$ and $\left(v, w_{2}\right)$ for some $w_{1}, w_{2} \in V(H)$. Hence, $E$ has the form $v \times E_{H}^{\prime}$ for some edge $E_{H}^{\prime}$ of $H$. As $A_{H}^{\prime}$ is a 2-infection set, $E_{H}^{\prime}=E_{H}$, and our first claim follows.

Next we will show that $A_{1}$ infects all the vertices $(g, h)$ where $g \notin A_{G}$. Let $E_{G}$ be an edge of $G$ that is infected by a set $A_{G}^{\prime}$ (starting from $A_{G}$ ). We want to show that for a given $w \in V(H)$, the edge $E_{G} \times w$ is the only uninfected edge of $G \square H$ that contains $A_{G}^{\prime} \times\{w\}$. If $A_{G}^{\prime}$ has size at least 2 , then this can be seen since it is similar to the first claim. If $A_{G}^{\prime}$ has size 1 , then we can assume that $(v, w)$ is the single element of $A_{G}^{\prime} \times\{w\}$ (where $v \in V(G)$ ). The only edges of $G \square H$ containing $(v, w)$, other than $E_{H} \times w$, must have the form $v \times E_{H}$ for some edge $E_{H}$ of $H$. By our first claim, the edge $E_{H}$ is already infected. This proves the assertion. 
Notice that we have $I_{2}(H)=V(H)$ if $H$ is a graph, so our result implies Equation (1). Lemma 6.9 and Theorem 6.10 imply the following bound that avoids the concept.

Corollary 6.11. Let $G$ and $H$ be two hypergraphs, then

$$
I(G \square H) \leq I(G)(I(H)+|E(H)|) .
$$

This bound can be tight, e.g. when $G$ and $H$ each consist of one single edge of size at least 2 .

\section{$7 \quad$ Line graphs}

In Section 1 the line graph of a hypergraph is defined. It is possible to construct a hypergraph $H$ for any graph $G$ such that $L(H)=G$. Simply let the vertices of $H$ be the edges in $G$ and for each vertex of $G$ let the set of all edges incident to the vertex be an edge in $H$. Call this the adjacency hypergraph for $G$.

Lemma 7.1. For any connected simple graph, the infection number of the corresponding adjacency hypergraph is 2 .

Proof. Let $G$ be a connected simple graph and $H$ the adjacency hypergraph of $G$. Each vertex in $H$ has degree 2 since it corresponds to an edge in $G$ with two end points. Further a pair of vertices in $H$ can belong to at most one edge, since otherwise they would corresponds to a double edge in $G$.

Thus any two adjacent infected vertices in $H$ can infect the edge in which they are contained. Then the remaining vertices in the edge occur in only one other edge each. These vertices can then force the other edges that contain them. Since $G$ is connected, continuing like this the hypergraph $H$ can be infected by any two adjacent vertices.

It has been shown in [5. Theorem 3.11] that for any graph $G$

$$
Z(G) \leq 2 Z(L(G))
$$

We state the following lemma, but we suspect that a stronger result holds.

Lemma 7.2. For any $k$-hypergraph $H$,

$$
I(H) \leq k Z(L(H)) .
$$

Proof. For $v$ in the vertex set of $L(H)$, let $E_{v}$ be the corresponding edge in $H$. Let $Z$ be a zero forcing set for $L(H)$. Add all the vertices of $H$ that are in the edge $E_{v}$ where $v \in Z$ to a set $A$. We claim that $A$ is an infection set for $I(H)$ of size $k Z(L(H))$.

Assume that in the first step of the zero forcing process on $L(H)$ (using the set $Z$ ) that $v$ forces $w$. Then all the vertices in $E_{v}$ are initially infected, further, all the vertices in any edge intersecting $E_{v}$ are also all infected, except the vertices in $E_{w}$. Thus the vertices in $E_{v} \cap E_{w}$ can infect $E_{w}$. Continuing like this, shows that $A$ is an infection set for $I(H)$. 


\section{Further work}

This paper is a first generalization of zero forcing to hypergraphs. There are many open problems and different directions in which to continue this work. A few are listed below.

We have seen that there are many different hypergraphs with infection number 1 , including any hypergraph in which the vertex set is an edge. It is also clear that if the infection number of a reduced hypergraph is 1 , then that hypergraph must have a degree 1 vertex. But this is in no way a sufficient condition for a hypergraph to have infection number 1 . It would be interesting to find some characterization of the $k$-hypergraphs that have infection number equal to 1.

Similarly, a graph on $n$ vertices has zero forcing number $n-1$ if and only if it is a complete graph. We would like to be able to characterize the $k$-uniform hypergraphs on $n$ vertices with infection number $n-(k-1)$. To this end, we make the following conjecture.

Conjecture 8.1. Assume that $H$ is a $k$-uniform hypergraph on $n$ vertices with $I(H)=n-(k-1)$. Then for any infection set of size $n-(k-1)$, the set of $k-1$ uninfected vertices are contained in an edge of $H$.

The zero forcing number for a tree has been well-studied [6]. In fact, it has been shown that the zero forcing number of a tree is equal to the minimum number of paths needed to cover the edges of the tree. It would be interesting to have an analogous result for hypertrees.

The first step is to determine what the appropriate hypergraph version of a tree is; one definition uses the notion of a host graph. For a hypergraph $H$, a host graph is a graph on the same vertex set as $H$ with the property that the vertices in any edge of $H$ induce a connected subgraph in the host graph. A hypergraph is called a hypertree if there exists a host graph of the hypergraph that is a tree.

So far our results in this direction are unsatisfying. For example, if $H$ is a hypertree, then it may happen that $I(H)>Z(T)$ for a host tree of $H$. To see this consider the hypergraph

$$
H:=\{(1,5,6),(2,5,6),(3,5,6),(4,5,6)\} .
$$

The tree below is a host tree for $H$

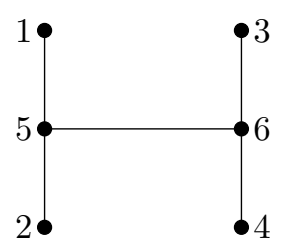

Note that $I(H)=3$; the set $\{1,2,3\}$ can infect the hypergraph, and there is no smaller set that forms an infection set. Also, the zero forcing number for the host tree above is 2 , since $\{1,3\}$ is a zero forcing set. In fact, we can make this difference arbitrarily large. Let $H=\left\{E_{1}, E_{2}, \ldots, E_{p}\right\}$ be a hypergraph on 
$n$ vertices that is a flower with $\left|E_{i} \cap E_{j}\right|=1$, then the graph $K_{1, n-1}$ is a host tree for $H$. In this case,

$$
p-1=I(H)<Z\left(K_{1, n-1}\right)=n-2 .
$$

The graph formed by taking the vertex sum of $p$ paths $P_{i}$, each with length $\left|E_{i}\right|-1$ is also a host graph for $H$. The zero forcing number of this graph is $p-1$.

We propose two questions about hypertrees. First, is it possible to determine a formula for the infection number of a hypertree or for a subfamily of hypertrees? Second, is there a relationship between zero forcing of a host tree and infection number of hypertree?

If $2 t+1 \geq k$ and $n \leq 2 t$, then we are not aware of a formula for the infection number for $C_{n}^{(k)}(t)$. We would like have a more complete picture for infection of hypercycles.

Finally, we have defined $m$-infection, this concept is useful for Cartesian products. It would be interesting to determine the $m$-infection number for some families of graphs and to find other applications of the concept.

Acknowledgments. The work in this paper was a joint project of the Discrete Mathematics Research Group at the University of Regina, attended by all the authors.

\section{References}

[1] Francesco Barioli, Wayne Barrett, Shaun M. Fallat, H. Tracy Hall, Leslie Hogben, Bryan Shader, P. van den Driessche, and Hein van der Holst. Zero forcing parameters and minimum rank problems. Linear Algebra Appl., 433(2):401-411, 2010.

[2] Francesco Barioli, Wayne Barrett, Shaun M. Fallat, H. Tracy Hall, Leslie Hogben, Bryan Shader, P. van den Driessche, and Hein van der Holst. Parameters related to tree-width, zero forcing, and maximum nullity of a graph. J. Graph Theory, 72(2):146-177, 2013.

[3] Csilla Bujtás, Michael A. Henning, and Zsolt Tuza. Transversals and domination in uniform hypergraphs. European J. Combin., 33(1):62-71, 2012.

[4] Charles J. Colbourn and Jeffrey H. Dinitz, editors. Handbook of combinatorial designs. Discrete Mathematics and its Applications (Boca Raton). Chapman \& Hall/CRC, Boca Raton, FL, second edition, 2007.

[5] Linda Eroh, Cong X. Kang, and Eunjeong Yi. Metric dimension and zero forcing number of two families of line graphs. Math. Bohem., 139(3):467483, 2014.

[6] AIM Minimum Rank-Special Graphs Work Group. Zero forcing sets and the minimum rank of graphs. Linear Algebra Appl., 428(7):1628-1648, 2008.

[7] Leslie Hogben, My Huynh, Nicole Kingsley, Sarah Meyer, Shanise Walker, and Michael Young. Propagation time for zero forcing on a graph. Discrete Appl. Math., 160(13-14):1994-2005, 2012. 
[8] Liang-Hao Huang, Gerard J. Chang, and Hong-Gwa Yeh. On minimum rank and zero forcing sets of a graph. Linear Algebra Appl., 432(11):29612973, 2010.

[9] Simone Severini. Nondiscriminatory propagation on trees. J. Phys. A, 41(48):482002, 10, 2008.

[10] Boting Yang. Fast-mixed searching and related problems on graphs. Theoret. Comput. Sci., 507:100-113, 2013. 